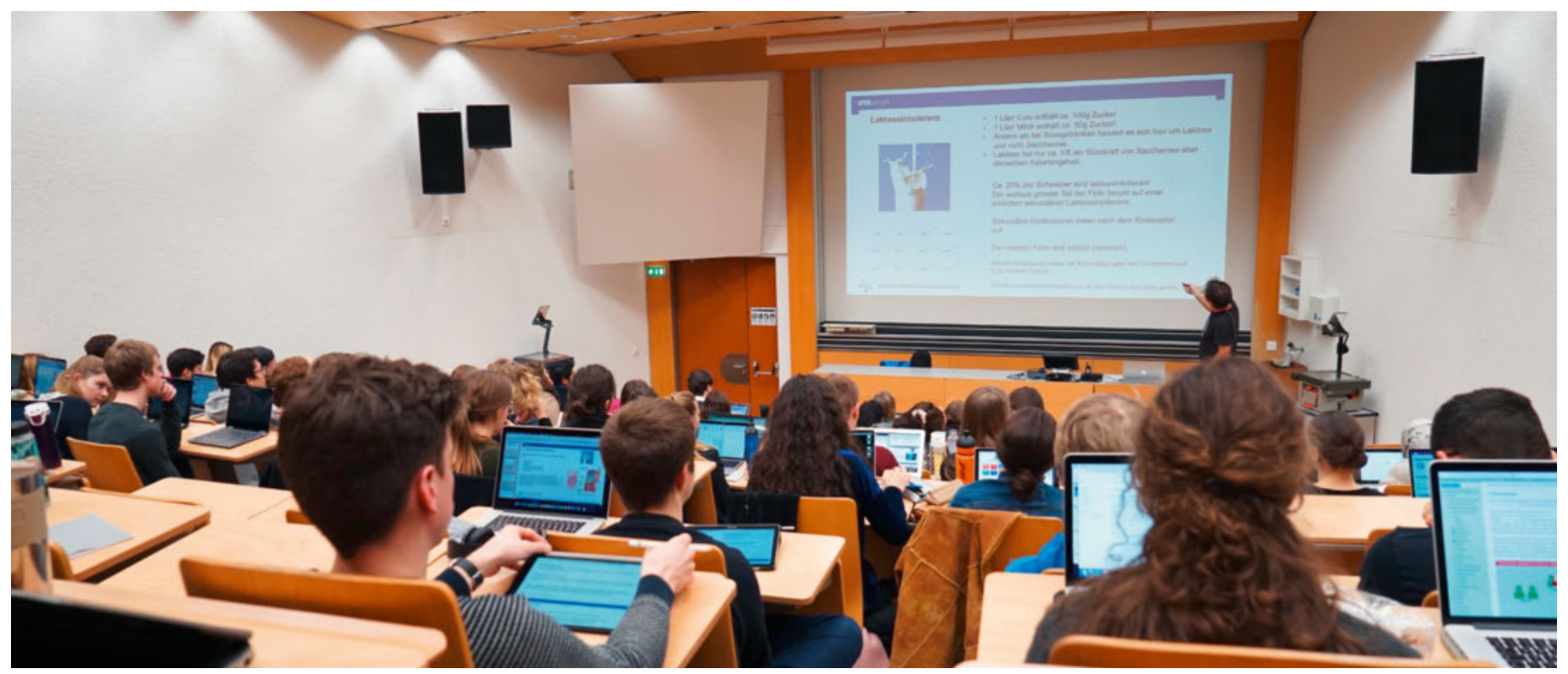

\title{
"Wir haben das Privileg, auf der grünen Wiese zu bauen»
}

Matthias Scholer

Wissenschaftsjournalist

Im letzten Herbst begannen 100 Studierende das neu von der ETH Zürich angebotene Bachelorstudium in Humanmedizin. Ziel des Studienganges ist es, medizinische Themen mit technisch-naturwissenschaftlichen Aspekten zu verbinden und die angehenden Ärztinnen und Ärzte auf die sich rasch ändernden Herausforderungen in diesem Bereich vorzubereiten. Aber auch Themen wie Empathie und Kommunikation kommen nicht zu kurz. Dank einem innovativen Konzept und modernsten Lernmethoden soll dieser Spagat gelingen.

Herr Goldhahn, wie waren die Reaktionen seitens der Universitäten, die schon länger ein Medizinstudium anbieten, als die ETH ihre Pläne für ein eigenes Bachelorstudium in Humanmedizin präsentierte? $\mathrm{Zu}$ Beginn war sicherlich eine gewisse Reserviertheit spürbar. Das hat sich jedoch zwischenzeitlich gelegt. Schliesslich sind wir ja auch nicht Konkurrenten, sondern ein neuer Player, der mithilft, die Ziele des Sonderprogrammes des Bundes zur Steigerung der Absolventenzahl eines Humanmedizinstudiums zu erfüllen. Speziell mit unseren Partneruniversitäten im
Tessin, in Basel und in Zürich pflegten wir von Anfang an eine enge Zusammenarbeit. Aber auch mit den anderen Institutionen tauschen wir uns regelmässig aus. Das Interesse an unseren Erfahrungen wächst, denn die ETH ist in gewisser Hinsicht ein neuartiger Player.

\section{Inwiefern?}

Zum einen bietet die ETH das Studium vor ihrem technisch-naturwissenschaftlichen Hintergrund an. Als neuer Anbieter haben wir aber auch die Chance, historisch gewachsene Aspekte in der Grundausbildung kri- 


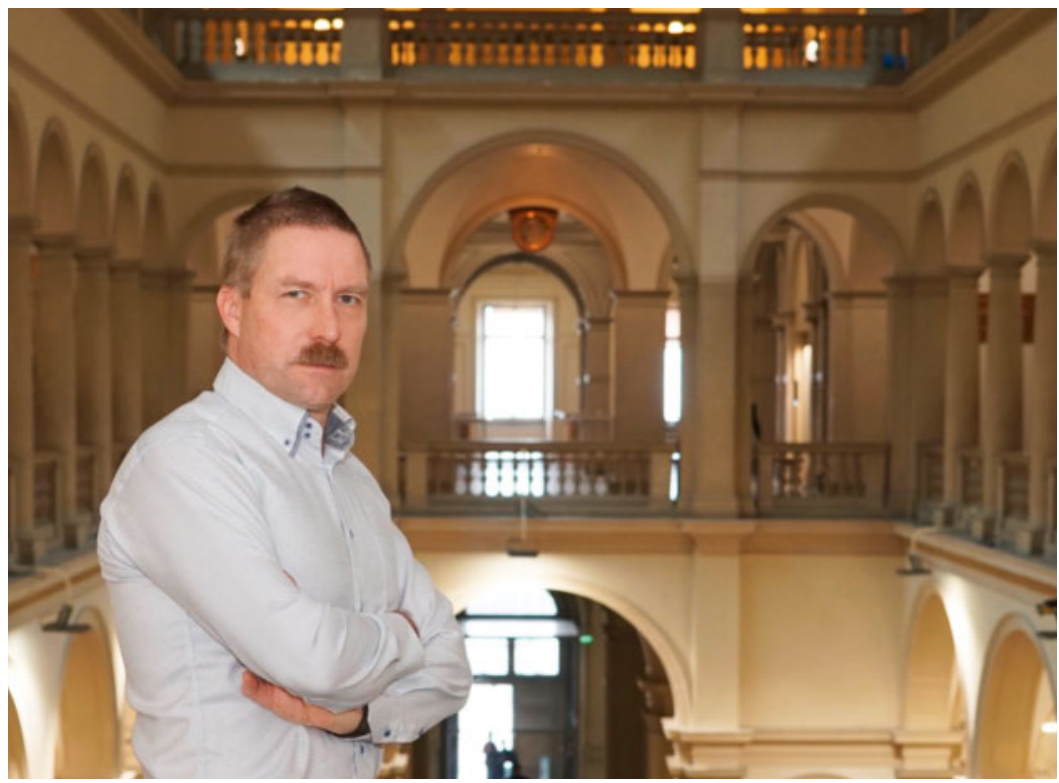

Jörg Goldhahn leitet das Projetteam «Bachelors Humanmedizin» an der ETH.

tisch zu hinterfragen. Wir haben das Privileg, "auf der grünen Wiese» zu bauen. Das stellt eine grosse Herausforderung, aber gleichzeitig auch eine einmalige

«Die meisten Studiengänge sind so aufgebaut, als ob man auf einer einsamen Insel sitzt und als Ärztin, als Arzt auf sich alleine gestellt Entscheide fällen muss.»

Chance dar - nicht nur für die ETH, sondern auch für die Studierenden. So sind diese die ersten, die nach den Vorgaben des neuen Schweizer Lernzielkatalogs ausgebildet werden, der Stoff wird ihnen zudem mittels modernster, wissenschaftlich geprüfter Lern- und Lehrmethoden vermittelt. Da gibt es kaum mehr klassischen Frontalunterricht, bei dem die Studierenden in einen passiven Survival Mode umschalten können.

\section{Können Sie das Curriculum kurz umschreiben?}

Neben der Vermittlung medizinischen Grundwissens haben wir uns drei Schwerpunkte gesetzt: Erstens wollen wir die Chancen und Risiken, welche die rasch fortschreitende Technisierung in der Medizin mit sich bringt, thematisieren. Zweitens müssen die zukünftigen Ärztinnen und Ärzte kompetent mit der enormen Informationsflut, der sie ausgesetzt sind, umgehen können. In diesem Bereich sehe ich grossen Aufholbedarf. Denn die Vostellung, dass man als Ärztin oder Arzt auf sich alleine gestellt Entscheide fällen muss, entspricht nicht der Realität. Ohne Internetrecherchen und einen intensiven Erfahrungsaus- tausch unter Kollegen werden heute kaum mehr komplexe Entscheidungen gefällt. Diesem Umstand muss man bereits im Grundstudium Rechnung tragen. Deshalb vermitteln wir unseren Studierenden nicht nur den Umgang mit Patienten, die sich im Internet über ihre Krankheit vorinformiert haben. Die angehenden Ärztinnen und Ärzte lernen auch, wie sie verschiedene Portale und Datenbanken nutzen können, um dank wissenschaftlich fundierter Informationen den Überblick über die neusten Erkenntnisse auf ihrem Fachgebiet behalten zu können.

\section{Und der dritte Schwerpunkt?}

Ist die Arbeit in Fach und Profession übergreifenden Teams. Eine Tendenz, die nicht mehr aus der heutigen Medizin wegzudenken ist. Denken wir nur an Stroke Units oder interdisziplinäre geriatrische Abteilungen. Die Vorteile einer solchen Teamarbeit wollen wir unseren Studierenden von Beginn weg aufzeigen und so vermeiden, dass sie in hierarchisches Denken fallen.

\section{Aber medizinische Grundlagen wie Anatomie und Nomenklatur werden schon noch vermittelt?}

Selbstverständlich. Sie bilden das Grundgerüst des Studienganges. Ohne eine gehörige Portion medizinischen Allgemeinwissens geht es nicht. Bei einem solch breit aufgestellten Curriculum kommt es jedoch unweigerlich zum Zielkonflikt «Vollständigkeit versus Verständnis». Wir legen beispielsweise weniger Wert darauf, dass unsere Studierenden unzählige Krankheitsbilder auswendig lernen. In dieser Zeit sollen sie lieber den Umgang mit Diagnose-Assistenzsystemen trainieren.

\section{Die ETH hat traditionell eher eine mathematisch- technologische Prägung. Ist es folglich auch das Ziel der ETH, angehende Medizinerinnen für eine Karriere in der Forschung vorzubereiten?}

Lassen Sie mich es anders ausdrücken: Wir wollen an Forschung interessierte Mediziner ausbilden. Tatsächlich beinhaltet unser Studiengang als einziger in der Schweiz während zwei Semestern Mathematik als Studienfach. Wir sind überzeugt, dass ein profundes

«Wir sind überzeugt, dass ein profundes mathematisches Wissen künftig auch im Arztberuf eine zentrale Rolle spielen wird.»

mathematisches Wissen künftig auch im Arztberuf eine zentrale Rolle spielen wird. Sei es beispielsweise bei der Interpretation von Statistiken oder dem algorithmischen Denken beim Umgang mit Applikationen. 
Das tönt alles sehr technikorientiert. Braucht eine gute Ärztin, ein guter Arzt nicht auch Qualitäten wie Empathie und ausgeprägte kommunikative Fähigkeit?

Selbstverständlich. Wir messen diesen Themen viel Bedeutung zu und haben entsprechende Kurse vom ersten Semester an im Curriculum eingebaut. So absolvieren unsere Studierenden nicht nur ein Pflegeprakti-

\section{«Später kommen weitere vertiefende Kurse, zum Beispiel im Bereich des Shared Decision Making, hinzu."}

kum, um die menschliche, pflegerisch-fürsorgliche Seite kennenzulernen. Sie lernen und üben bereits im ersten Semester den Umgang mit Patienten und Angehörigen. Später kommen weitere vertiefende Kurse, zum Beispiel im Bereich des Shared Decision Making, hinzu. Dabei müssen die Studierenden einen fiktiven Patienten über seine Therapieoptionen, deren Vor- und Nachteile beziehungsweise Risiken informieren, um dann gemeinsam mit ihm und unter Einbezug aller Fachmeinungen einen Therapieentscheid zu fällen. Diese Gespräche werden von einer Ethikerin, einer Journalistin und einer Juristin verfolgt. Abschliessend beurteilen diese das Vorgehen der Studierenden. Die Ethikerin bewertet dabei die gemachten Aussagen aus ihrer Warte, während sich die Journalistin auf die

\section{Zur Person}

Jörg Goldhahn studierte in Jena Medizin und absolvierte anschliessend an der ETH den Nachdiplomstudiengang "Medical Physics». Danach leitete Jörg Goldhahn an der ETHZ die Forschungsgruppe "Biomechanics of Ageing", bevor er während fünf Jahren bei Novartis als Direktor «Translational Medicine» tätig war. Ende 2017 wechselte Goldhahn zur ETHZ zurück, wo er einerseits als Vizedirektor dem Institute for Translational Medicine vorsteht und andererseits das Projektteam ETH-Bachelor in Humanmedizin leitet.

Kommunikation fokussiert und die Juristin die rechtlichen Aspekte abdeckt. Soweit ich weiss, gibt es bislang noch keinen vergleichbaren Kurs an einer anderen Hochschule.

\section{War es schwierig, Dozierende zu finden?}

Im Gegenteil. Als die Pläne und der Studienaufbau konkret wurden, meldeten sich viele Expertinnen und Experten, die gerne ein Teil davon werden wollten. Sie schätzen vor allem, dass bei unserem Studiengang die Interaktivität zwischen den Dozierenden und Studierenden im Vordergrund steht. Wir sind aufgrund der Erfahrungen im ersten Studienjahr hocherfreut, wie aktiv sich die Studierenden am Unterricht beteiligen.

Bildnachweis

Matthias Schole 